JAFIB'

Journal Review

\title{
Journal of Atrial Fibrillation
}

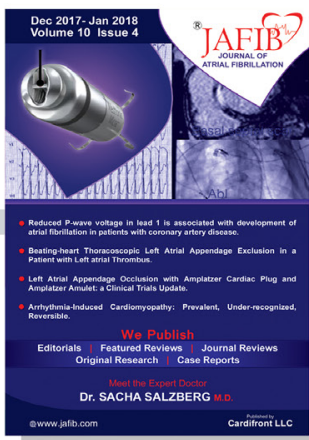

wWW. jafib.com

\section{Management of Stroke Risk in Atrial Fibrillation Patients with Bleeding on Oral Anticoagulation Therapy-Role of Left Atrial Appendage Closure, Octreotide and more}

\author{
Tawseef Dar ${ }^{1}$, Bharat Yarlagadda ${ }^{1}$, James Vacek, Buddhadeb Dawn, Dhanunjaya Lakkireddy ${ }^{1}$ \\ ${ }^{1}$ Division of Cardiovascular Diseases, Cardiovascular Research Institute, University of Kansas Hospital E Medical Center, \\ Kansas City, KS.
}

\begin{abstract}
Background: Bleeding complications especially gastrointestinal bleeding remains a major challenge associated with oral anticoagulation therapy (OAT) and often leads clinicians to withdraw oral anticoagulation therapy (OAT). This exposes patients to risk of stroke and systemic thromboembolism (STE). Novel oral anticoagulants (NOACs) have proved no better when it comes to bleeding events and in fact studies have shown that overall NOACs are associated with higher incidence of gastrointestinal (GI) bleeding compared to warfarin [4].

Objectives: In this review, we describe the difficulties encountered in managing OAT in patients with bleeding and strategies to maneuver around these bleeding complications particularly gastrointestinal bleeding secondary to arteriovenous malformations (AVM) and other vascular abnormalities.

Findings: Left atrial appendage closure (LAAC) has emerged as a very elegant and promising tool for stroke prevention in non-valvular atrial fibrillation (AF) patients who are intolerant to OAT. But the need for OAT post procedure for a brief period is becoming a major hurdle for clinicians to pursue in this direction in patients with recurrent gastrointestinal bleeds. And in majority of cases, recurrent or refractory gastrointestinal bleeds are usually secondary to arteriovenous malformations/angiodysplasias (AVM/AD). We suggest that the problem has to be approached by decreasing or eliminating the acute bleeding risk and closing the LAA in the long term, to enable the patients to come off of OAT and minimize the risk of recurrent bleeding.

Conclusion: Recurrent GI bleeding, secondary to arteriovenous malformation (AVM), is one of the common reasons for OAT discontinuation in atrial fibrillation patients. Newer techniques of left atrial appendage closure (LAAC) offer some respite in such cases but we are in an era of transition wherein different options available for stroke prevention in atrial fibrillation patients are still interdependent on each other and therefore role of drugs like Octreotide (OCT) and other similar agents like steroids, hormonal therapy etc. becomes especially important and worth a trial in such cases. While we continue to work for future atrial fibrillation patients we should not forget about atrial fibrillation patients at present.
\end{abstract}

\section{Introduction}

Atrial fibrillation (AF) continues to remain a major cause of morbidity and mortality and a big challenge for clinicians around the globe for a variety of reasons. The most important being the stroke risk associated with atrial fibrillation, which tends to be more severe in patients with atrial fibrillation $(\mathrm{AF})$ than in patients without $\mathrm{AF}^{[1]}$. At the same time the risk of bleeding especially life threatening intracranial hemorrhage $(\mathrm{ICH})$ and gastrointestinal (GI) bleeding associated with oral anticoagulation therapy (OAT) significantly increases with OAT. And ironically the incidence of $\mathrm{AF}$, the risk of bleeding and the incidence of strokes attributable to atrial fibrillation all increase with the age, making it a particu-

Key Words

Atrial fibrillation, Left Atrial Appendage, Arteriovenous malformations, Angiodysplasias, Octreotide.

\section{Corresponding Author}

Dhanunjaya Lakkireddy

Professor of Medicine Division of Cardiovascular Diseases, Cardiovascular Research Institute,

University of Kansas Hospital and Medical Center, 3901 Rainbow Boulevard Kansas City, Kansas 6616. larly difficult situation ${ }^{[2]}$. As for the above fact and the aging of our population, it is estimated that by $2020,7.5$ million individuals will have AF in the United States alone ${ }^{[3]}$.

Novel oral anticoagulant (NOACs), despite having emerged as safer and more effective or equal alternative to vitamin $\mathrm{K}$ antagonists (VKA), still continue to have bleeding complications. The ground reality is that the risk of bleeding while being anticoagulated is always there for the simple fact that risk for both the thromboembolism and the bleeding are largely driven by an overlapping set of comorbidities [4]. Approximately $50 \%$ of patients with AF who have guideline indication for oral anticoagulation therapy (OAT) end up not being on any form of oral anticoagulation therapy for various reasons with bleeding being the most common and obvious reason. ${ }^{[5]}$. This leads to a large population of AF patients who remain at risk for preventable stroke. Stroke is the leading cause of disability and third leading cause of death in United States with an estimated annual cost of around 60 billion dollars ${ }^{[6]}$. Left atrial appendage closure (LAAC) has offered some respite in a number of such patients in the long term. The LAAC devices have been used with varying OAT and 
Antiplatelet Therapy(APT) protocols in different parts of the world with varied experiences. However, randomized controlled trials for LAAC have been done using intra and post-procedural OAT for 6 weeks and dual APT for 6 months. This presents a huge hurdle in pursuing endocardial LAAC in patients suffering from bleeding complications. Epicardial ligation or clipping of the LAA has been done with great efficacy with no need for post procedural OAT but has not been studied in a RCT.

Prevention of Thromboembolic phenomena in atrial fibrillation patients-Road bumps and detours-a brief background

OAT continues to remain the first line of therapy for prevention of thromboembolic events in patients suffering from AF. Current AHA/ACC/HRS guidelines recommend use of OAT in all AF patients with CHADSVaSc score greater or equal to 2, regardless of cardioversion/ablation or type of $\mathrm{AF}^{[7]}$.

Warfarin with target INR of 2-3

It has traditionally been and is still being used for prevention of thromboembolic phenomena in AF patients with much success. However, bleeding complications associated with warfarin use have always been an issue. Typical bleeding rates reported in randomized trials are in the range of 1 to 3 percent per person-year, which tends to be an underestimation of the actual bleeding rates in practice. One of the recent studies showed that the rate of nonfatal and fatal bleedings for warfarin monotherapy were 3.6 and 0.2 percent per patient-year respectively with GI bleed accounting for a significant proportion of nonfatal bleeding events ${ }^{[8]}$. The bleeding rate significantly goes up when warfarin is combined with antiplatelet agents [Table 1].

Lowering target INR has been suggested in the past to offset the bleeding risk but studies did not show any effect on hemorrhage rate with such strategy. Low intensity warfarin was found to be less efficacious than conventional intensity warfarin with no reduction in

\begin{tabular}{|c|c|c|c|c|}
\hline & \multicolumn{4}{|c|}{$\begin{array}{l}\text { \%per Patient-year } \\
\text { Variable }\end{array}$} \\
\hline & $\begin{array}{l}\text { Warfarin } \\
\text { Monotherapy }\end{array}$ & $\begin{array}{l}\text { Warfarin+ } \\
\text { Aspirin }\end{array}$ & $\begin{array}{l}\text { Warfarin+ } \\
\text { Clopidogrel }\end{array}$ & $\begin{array}{l}\text { Warfarin+ } \\
\text { Aspirin+ } \\
\text { Clopidogrel }\end{array}$ \\
\hline Nonfatal bleeding & 3.6 & 6.4 & 13.3 & 15.4 \\
\hline Fatal bleeding & 0.2 & 0.4 & 0.6 & 0.2 \\
\hline $\begin{array}{l}\text { Fatal and nonfatal } \\
\text { bleeding }\end{array}$ & 3.9 & 6.8 & 13.9 & 15.7 \\
\hline Intracranial bleeding & 0.6 & 0.8 & 0.8 & 1.0 \\
\hline Airway bleeding & 1.3 & 2.3 & 7.1 & 7.1 \\
\hline $\begin{array}{l}\text { Gastrointestinal } \\
\text { bleeding }\end{array}$ & 0.9 & 2.1 & 3.8 & 5.1 \\
\hline Urinary tract bleeding & 1.0 & 1.6 & 2.0 & 2.4 \\
\hline
\end{tabular}

the risk of clinically significant bleeding over time ${ }^{[9]}$. Low intensity fixed dose warfarin plus Aspirin was also studied but was found to be less efficacious than adjusted dose warfarin in preventing stroke in high risk patients ${ }^{[10]}$. Aspirin and Plavix combination was more effective than aspirin alone but less effective than warfarin ${ }^{[11]}$. Subcutaneous Idraparinux was also studied and was found to be more efficacious than warfarin but at the cost of substantially higher risk of bleeding ${ }^{[12]}$. Ximelagatran, a direct thrombin inhibitor, had equal safety and efficacy as warfarin but was found to be hepatotoxic ${ }^{[13]}$. Drug and food interactions, unpredictable daily variations in the INR, need for periodic checks has made warfarin relatively unpopular with both patients and physicians.

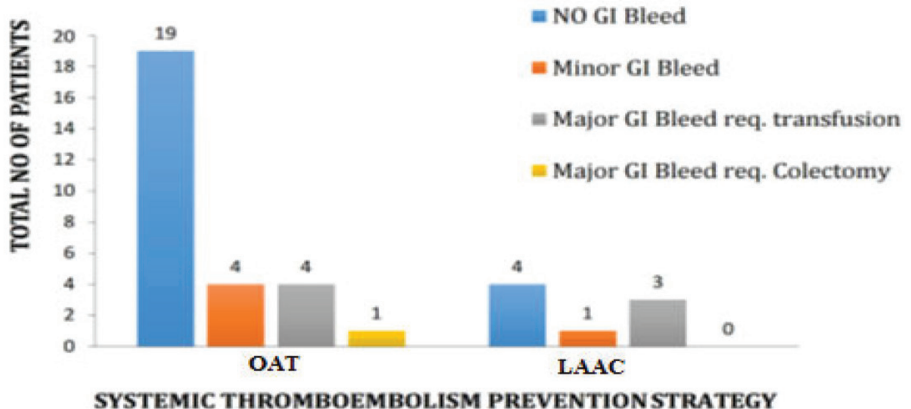

Figure 1:

Showing the impact of OCT therapy on GI bleeding in patients on OAT

Novel Oral Anticoagulant agents (NOAC)

Continuous efforts to mitigate the problems of warfarin lead us into an era of Novel Oral anticoagulants. NOAC's target different parts of the coagulation pathway, have a more predictable anticoagulant effect, and do not require INR monitoring. This group comprises of direct thrombin inhibitors (e.g. Dabigatran) and direct factor Xa inhibitors (apixaban, rivaroxaban, edoxaban). Although NOACs were very effective in averting many of the downsides of warfarin like drug interactions, INR monitoring, unpredictability of anticoagulation, risk of bleeding while on NOACs stayed there. Studies showed that, in comparison to warfarin, apixaban was associated with lower risks of major bleeding, intracranial bleeding and gastrointestinal bleeding, Dabigatran was associated with lower risks of major bleeding and intracranial bleeding with no significant difference in the risk of gastrointestinal bleeding and Rivaroxaban was associated with similar risk of major bleeding but lower risk of intracranial bleeding and higher risk of gastrointestinal bleeding [Table 2] ${ }^{[14]}$. Overall, in comparison to warfarin, NOACs lower the risk for intracranial bleeding and probably also decrease the overall risk for major bleeding episodes but surprisingly increase the risk for gastrointestinal bleeding in atrial fibrillation patients ${ }^{[15]}$. And, with no FDA approved antidotes available except for Dabigatran, the issue of bleeding with NOACs got even more complicated especially for patients leading to non-compliance. Also, with no data for their use in the presence of mechanical heart valves or Valvular atrial fibrillation, a significant proportion of patients with atrial fibrillation are still dependent on warfarin for stroke prevention.

Despite substantial evidence about the risk of bleeding with NOACs, clinicians have been using them in patients who bled on warfarin as an alternative therapy without much success for obvious reasons. A recent multicenter real world study published by our group showed NOACs may not be all that much better in minimizing bleeding complications in warfarin ineligible patients. 265 warfarin ineligible patients who were started on NOACs were followed for a mean duration of $14 \pm 4$ months. Repeat major bleeding events (MBE) occurred in $63 \%$ of patients and incidence was significantly higher in patients with prior history of GI bleeding $(74.5 \%$ vs. $30 \%$, $\mathrm{P}<0.0001)$ than those without. HAS-BLED score, type of NOACs used or concomitant aspirin or clopidogrel use had no impact on repeat major bleeding event (MBE). However, subgroup analysis of the most challenging group of patients with left atrial appendage thrombus and intracranial hemorrhage on warfarin treated with a NOAC came up with some reassuring figures.13 out of 14 such patients had resolution of clot within 3 months but $50 \%$ developed a 
Table 2: Comparison of NOACs vs Warfarin in terms of bleeding

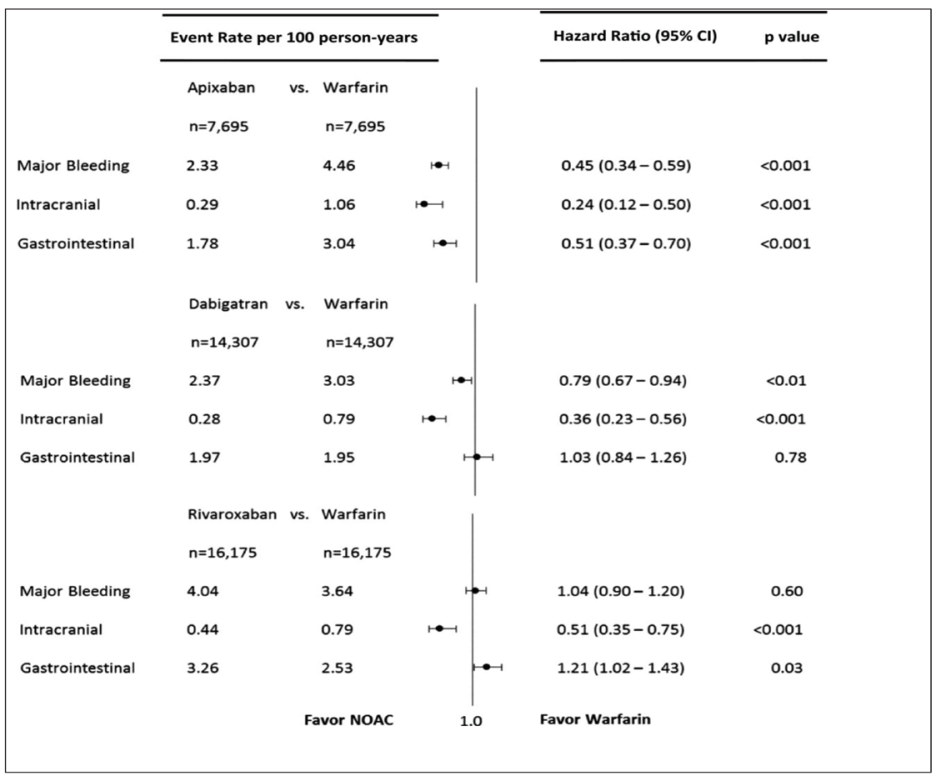

Reproduced from Journal of the American Heart Association. 2016;5:e003725

repeat intracranial hemorrhage (ICH) or systemic thromboembolism (STE) on a NOAC. In patients who did not have another event $(50 \%$ -7 of 14), majority of them $71 \%$ (five of seven) underwent successful left atrial appendage occlusion device implantation followed by temporary bridging with $\mathrm{NOACs}$ during the periprocedural period and $29 \%$ (two of seven) were continued on a NOAC with no further adverse events ${ }^{[4]}$.

So the bottom line is that the risk of bleeding is always there no matter which anticoagulant is used and this leads to a large proportion of atrial fibrillation patients not being on any kind of anticoagulation despite having guidelines indications for the same. In this article we will review the strategies to maneuver around these bleeding complications and managing them.

Role of Left atrial appendage closure (LAAC) in stroke prevention and mitigating bleeding complications

Left atrial appendage closure (LAAC) has evolved to be a very promising solution in many of these patients who are high risk for long-term use but eligible for OAT. The PROTECT-AF and PREVAIL-AF studies have clearly shown the cumulative advantage of a LAAC over warfarin for major bleeding, stroke and death together. The benefit is more profound in mitigating the risk of major bleeding (including ICH and GI bleeding) and mortality. The stroke benefits are non-inferior to warfarin ${ }^{[16]}$. It is obvious that the warfarin group did exceptionally better in the study setting than what ones sees in a real-world setting. The ability of an OAT in preventing a LA clot and subsequent STE is expected to be superior to any other approach. However the associated bleeding risk is what makes these agents undesirable. At least in the current medical armamentarium there is no agent that can prevent stroke without the risk of bleeding. This exactly where LAAC devices fit in. Off label use of endoepicaridal suture with the Lariat device and Atriclip has been seen in several OAT contraindicated patients.

Small registries on Watchman, Amplatzer Cardiac Plug and Lariat have shown 2-4 fold reduction in stroke rates in OAT contraindicated patients compared to their corresponding $\mathrm{CHADS}_{2}$ score based stroke risk. The use of LAAC without post procedural OAT has been widely prevalent. It is probably hard to conduct a $\mathrm{RCT}$ in this specific subset of population. There are several larger ongoing registries that are addressing this specific issue. FDA approved the Watchman device in the United States in 2015 and has slowly gained momentum in clinical use. Current AHA/ASA Guidelines recommendations suggest closure of the left atrial appendage (LAA) for high-risk patients with AF who are deemed unsuitable for anticoagulation if performed at a center with low rates of periprocedural complications and the patient can tolerate the risk of at least 45 days of postprocedural anticoagulation (Class IIb; Level of Evidence B $)^{[17]}$. European Society of Cardiology recommends LAAC for patients with non-valvular AF, who are either contraindicated or Unsuitable for long tern OAT-owing to high bleeding risk (HASBLED score greater or equal to 3) Or as an alternative treatment (Class IIb; Level of Evidence B) ${ }^{[18]}$. European Heart Rhythm Association (EHRA) and European Association of Percutaneous Cardiovascular Interventions (EAPCI), recommends LAAC in patients with $\mathrm{AF}$ and indication for OAT for stroke/embolism prevention (with $\mathrm{CHA}_{2} \mathrm{DS}_{2}-\mathrm{VASc}$ score $>1$ point) and increased risk of bleeding (HAS-BLED score 3 points or more), contraindications for OAT, or refusal of treatment with OAT ${ }^{[19]}$. But the limitations of this intervention are that it can only be done in patients with non-valvular atrial fibrillation and no other indication for OAT and also patient needs to be able to tolerate intra-procedure and post-procedure anticoagulation as per the protocol. This requirement of uninterrupted post-procedure antithrombotic protocol for some months becomes a major hurdle in pursuing this procedure in patients at risk for bleeding.

Causes of gastrointestinal bleeding in atrial fibrillation patients on oral anticoagulation therapy (OAT)

The majority of atrial fibrillation patients are elderly with multiple comorbidities and are on multiple medications including antiplatelet therapy, NSAIDS, etc. leading to increased overall prevalence of mucosal erosions, gut dysmotility, peptic ulcer diseases, diverticulosis, polyps and arteriovenous malformations in this population. All these factors contribute to increased vulnerability of these patients to gastrointestinal bleeding while on OAT.

Arteriovenous malformations (AVM) are of particular importance due to varied reasons viz- accounting for $40-60 \%$ of lower and $5 \%$ of upper gastrointestinal bleeds respectively, often elusive, multiple and difficult to treat with conventional endoscopic methods ${ }^{[20]}$. Pathologically they are dilated, tortuous thin-walled communications between veins, venules and capillaries located in submucosa of the gut. They are usually located in small and large intestines and diagnostic studies include upper and lower endoscopies, wireless capsule endoscopy, double balloon enteroscopy and computed tomography (CT) or magnetic resonance angiography. Gastrointestinal bleeding has a huge impact on healthcare costs in US with mean cost/ hospital admission for lower GI blood loss being around $\$ 40,456^{[21]}$. Use of OAT in this group of patients is particularly challenging. Endoscopic cauterization, mesenteric arterial coiling or embolization, surgical resection of the affected regions may be reasonable options. But a large number of these patients continue to have repeat bleeding as more than one site is typically involved. Perhaps a systemic pharmacologic intervention to affect these AVMs may provide an opportunity to temporarily use OAT in these unfortunate patients with a long term plan for LAAC. 
Interventions for management of gastrointestinal bleeding in patients with atrial fibrillation

Non-pharmacologic interventions

The first line therapy for any patient with gastrointestinal bleeding on OAT is endoscopic intervention. This is both diagnostic and therapeutic except for capsule endoscopy (only diagnostic). If the source of bleeding is identified, it is usually subject to endoscopic therapy with argon plasma coagulation (APC), electrocoagulation or mechanical hemostasis using clips. Surgery with resection of the bowel segment, identified to be the source of bleeding, may be needed in life threatening hemorrhages. If no bleeding source is identified on endoscopies, angiography with embolization is usually the next step in management. Patients can subsequently be rechallenged with oral anticoagulation therapy. However advanced age and associated comorbidities in majority of atrial fibrillation patients makes these invasive interventions more challenging and risky. Above all, multifocal nature and inaccessibility of AVM lesions in some patients, results in overall recurrence rate of $30-40 \%$ despite all these aforementioned interventions ${ }^{[22]}$. And ultimately the end result is discontinuation of OAT.

\section{Pharmacologic Interventions}

Due to its recurrent nature and risks of invasive procedures in elderly patients, pharmacotherapy is particularly sought for lower gastrointestinal bleeding secondary to arteriovenous malformations $(\mathrm{AVMs})$. There is some evidence, mostly from anecdotal case reports, that supports the use of different pharmacological agents in such situations, as we describe further .Their overall benefits in isolated cases of lower gastrointestinal bleeding who are not on OAT is still a matter of debate. However, in patients with obligate need for OAT the overall clinical benefit outweighs the risks associated with those medications, as we showed in our study.

\section{Role of Octreotide}

Octreotide (OCT) is a long acting somatostatin analogue which acts as a vasoconstrictor and thereby decreases splanchnic blood flow. Octreotide has long been used in the management of acute variceal upper gastrointestinal bleed. Its role in non-variceal bleeds particularly angiodysplasias was less well understood until recent times. However, there have been multiple case reports, prospective non-randomized studies and a meta-analysis that showed its promising role in patients suffering from angiodysplasias ${ }^{[23]}$. Its role becomes particularly

\begin{tabular}{ll} 
Table 3: $\begin{array}{l}\text { Showing the OAT+OCT regimen used and improvement in mean Hb } \\
\text { level in patients with history of GI bleed on OAT }\end{array}$ & Patients \\
\hline \multicolumn{1}{c}{ Outcomes } & $15(53.5 \%)$ \\
Type of OAT Continued & $11(39.3 \%)$ \\
Apixaban & $02(7.1 \%)$ \\
Rivaroxaban & $09(32.1 \%)$ \\
Warfarin & \\
Recurrent of GI bleeding after restarting 0AT+0AC & $04(14.2 \%)$ \\
therapy & $04(14.2 \%)$ \\
Minor bleeds & $01(3.5 \%)$ \\
Major bleeding requiring transfusion & \\
Major bleeding requiring Colectomy & $7.49( \pm 1.07)$ \\
Hemoglobin(gm/dl) & $9.33( \pm 1.12)$ \\
At baseline & $11.10( \pm 1.50)$ \\
At 3 months follow up & \\
At 6 months follow up & \\
\hline Outcomes in patients on OAT/OCT therapy at 6 months follow $(\mathrm{n}=28)$
\end{tabular}

important in patients who have refractory bleeding, inaccessible or multiple lesions, patients at high risk for interventions and patients on anticoagulants/antithrombotic therapies. These vascular lesions represent a relatively common cause of gastrointestinal bleeding, especially among the elderly people. Because recurrent gastrointestinal (GI) bleeds and drop in hemoglobin due to these angiodysplasias forces clinicians to withdraw OAT, its role in patients with several co-morbidities and in need of anticoagulation becomes particularly noteworthy. One of the studies showed that use of long acting Octreotide (Octreotide LAR-Once a month IM injection) was associated with less bleeding events and transfusion needs with improvement in hemoglobin levels during octreotide treatment and interestingly enough most patients had severe co-morbidities and that treatment with either anticoagulants or antiplatelets was not suspended ${ }^{[24]}$. Octreotide can play a very vital role in atrial fibrillation patients who are off of OAT secondary to recurrent gastrointestinal (GI) bleeds or drop in hemoglobin level. It can help reinitiate OAT in many of these patients. Now that we are in an exciting era where we have options of LAAC, NOACs and 3D-mapping assisted radiofrequency ablation (RFA), role of octreotide can be particularly explored in atrial fibrillation patients. As mentioned above, recurrent gastrointestinal (GI) bleeding is one of the main hurdles in pursuing these procedures due to the fact that patient are still exposed to anticoagulation for a brief period after the procedure. Octreotide, with its impact on hemoglobin level and gastrointestinal (GI) bleeding recurrence, can help resume OAT in such patient and thereby pave the way for further available management options. Octreotide has an excellent safety profile with risk of gallstones, pancreatic diarrhea or impaired glucose metabolism in diabetics on long term use.

In a small prospective observational study [Figure 1], conducted by our group involving $36 \mathrm{AF}$ patients not on OAT due to recurrent gastrointestinal (GI) bleeds from AV malformations in the small and large intestine. These patients received 100 to $300 \mathrm{mg}$ of subcutaneous Octreotide injection twice daily and OAT was resumed 24-48 hours after OCT was started. The mean age was $69 \pm 8.0$ years with a mean $\mathrm{CHA}_{2} \mathrm{DS}_{2}$ VASc score of $3 \pm 1$ and the HASBLED score of $3 \pm$ 1. Before oral anticoagulation was discontinued, warfarin was used in $44 \%$, apixaban in $22.2 \%$, rivaroxaban in $16.7 \%$, and dabigatran in $16.7 \%$. LAA clot was present in $5 / 36(13.8 \%)$, and systemic thromboembolic events were reported in $8 / 36(22 \%)$ patients before enrollment. Angiodysplasia was the most common identified cause of bleeding (76\%) and one third of the patients (33.3\%) had a prior endoscopic intervention with APC of identifiable lesions, prior to enrollment. Median follow-up duration was 8 months (range of 6 - 13 months). Among the study population, 8/36 (22\%) patients underwent left atrial appendage closure for stroke prevention (LARIAT in 4, WATCHMAN in 3, and AtriClip in 1) with subsequent discontinuation of OAT and OCT therapy. But, while still on octreotide/anticoagulation therapy, 4/8 (50\%) had recurrent GI bleeds with 3 of them requiring blood transfusion [Figure 1].

The remaining 28/36 (77.8\%) patients were continued on oral anticoagulation with apixaban in $15(53.5 \%)$, rivaroxaban in 11 (39.3\%), and warfarin in 2 (7.1\%). Among these 28 patients, 19 (67.8\%) had no recurrent GI bleeds, while 4 (14.2\%) had minor GI bleeds, and 4 (14.2\%) had major GI bleeds requiring blood transfusion [Figure 1]. In those patients who remained on OAT throughout the study period $(\mathrm{n}=28)$, the mean hemoglobin levels were significantly higher at three months $(9.33 \mathrm{vs} .7 .49 \mathrm{~g} / \mathrm{dl} ; \mathrm{p}<0.001)$ 
and six months (11.10 vs. $7.49 \mathrm{~g} / \mathrm{dl} ; \mathrm{p}<0.001$ ) compared to baseline [Table 3], [Figure 2]. In addition, successful cardioversion was done in 13/36 (36\%) and were started on antiarrhythmic drugs (AAD) for maintenance of sinus rhythm and continued on oral anticoagulation along with octreotide. There were no reported events of systemic thromboembolism or intracranial hemorrhage during the study period. So overall, octreotide therapy enabled stroke prevention in patients with recurrent gastrointestinal bleeding by either continued oral anticoagulation use (78\%) or left atrial appendage closure (22\%). And $68 \%$ of patients who resumed and were maintained on oral anticoagulation therapy had no episode of recurrent gastrointestinal bleeding during the study period. No side effects from octreotide use were reported.

Potential role of other pharmacologic agents

The agents that have been studied the most in arteriovenous malformations (AVMs) are the hormones, estrogen and progesterone. There have been case series reports about successful use of estrogen in epistaxis but a randomized controlled trial (RCT), which included only 2 cases of gastrointestinal (GI) bleeding, failed to show any benefit ${ }^{[25]}$. Subsequently combination of estrogen and progesterone have also been studies in patient with gastrointestinal (GI) bleed

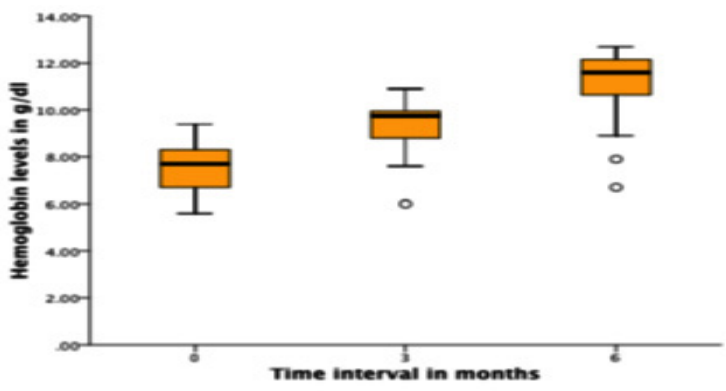

Figure 2:

Boxplot illustrating the improvement in mean $\mathrm{Hb}$ at 3 and 6 months post OCT therapy

secondary to AVM (AD and HHT). There have been multiple case reports about successful use of estrogen alone in doses ranging from $0.035 \mathrm{mg}$ to $0.05 \mathrm{mg}$, or estrogen $0.01 \mathrm{mg}$ to $0.1 \mathrm{mg}$ and progesterone $1 \mathrm{mg}$ to $5 \mathrm{mg}$ in patient with different AVM ${ }^{[26]}$. There was report of either bleeding stopped or reduction of transfusion requirements and an increase in hemoglobin for the duration of follow-up in most of these case reports. Subsequently two crossover trials both showed a significant impact on transfusion requirements in patients with AVM with combination hormonal therapy ${ }^{[27],[28]}$. One of the most recent Randomized double blind trial also failed to show any benefit of hormonal combination therapy over placebo ${ }^{[29]}$. However critics say that they used the lowest dose of estrogen reported so far which might have had an influence on the study outcome ${ }^{[30]}$. So overall, given the heterogeneity of results from different studies, there are no clear cut guidelines regarding efficacy of combination hormonal therapy in patients with AVM related gastrointestinal (GI) bleeding. Use of other pharmacologic agents like Prednisone, Danazol, Tranexamic acid (TA), Epsilon aminocaproic acid (EACA), Desmopressin, in patients with AVM related GI bleed, is mostly available from case reports [Table 4].

Interestingly enough, thalidomide-originally an anticancer drug, which made a comeback with its usefulness in Crohns disease, has also been reported to be useful in gastrointestinal (GI) bleeding related to angiodysplasias (AD). At low doses (100 mg/day to $200 \mathrm{mg} /$ day),

\begin{tabular}{|c|c|c|c|}
\hline $\begin{array}{l}\text { Table 4: } \begin{array}{l}\text { Pharmacolc } \\
\text { malformatic }\end{array}\end{array}$ & $\begin{array}{l}\text { gical agents u } \\
\text { ns }\end{array}$ & sed for treatr & ent of vascular \\
\hline Drug & $\begin{array}{l}\text { Predominant } \\
\text { diagnosis }\end{array}$ & $\begin{array}{l}\text { Route and } \\
\text { dose }\end{array}$ & Putative mechanism \\
\hline Estrogen $(E)$ & $\mathrm{AD} / \mathrm{HHT}$ & $\begin{array}{l}0.035 \\
\mathrm{mg}-0.05 \\
\mathrm{mg} \text { po }\end{array}$ & $\begin{array}{l}\text { Vascular stability, improved } \\
\text { coagulation, decreased } \\
\text { mesenteric blood flow }\end{array}$ \\
\hline$E+$ progesterone $(P)$ & $\mathrm{AD} / \mathrm{HHT}$ & $\begin{array}{l}0.01 \mathrm{mg}-0.1 \\
\mathrm{mg} \text { po }(\mathrm{E}) \\
1 \mathrm{mg}-2 \mathrm{mg} \\
\text { po }(\mathrm{P})\end{array}$ & $\begin{array}{l}\text { Vascular stability, improved } \\
\text { coagulation, decreased } \\
\text { mesenteric blood flow }\end{array}$ \\
\hline Octreotide & $\begin{array}{l}\text { A D / H H T, } \\
\text { BRBNS }\end{array}$ & $\begin{array}{l}100 \mathrm{mg}-500 \\
\mathrm{mg} \mathrm{sc} \text { bid, } 10 \\
\mathrm{mg}-30 \mathrm{mg} \mathrm{im}\end{array}$ & Multiple effects \\
\hline Corticosteroids & ws & po & Increased vascular integrity \\
\hline $\begin{array}{l}\text { Prednisolone } \\
\text { cyclophosphamide }\end{array}$ & ws & po & Increased vascular integrity \\
\hline Interferon & Hemangiomas & sc & Inhibits angiogenesis \\
\hline Danazol & HHT & $200 \mathrm{mg}$ po tid & $\begin{array}{l}\text { Weak androgen, direct vascular } \\
\text { stability }\end{array}$ \\
\hline Tranexamic acid & HHT & $1 \mathrm{~g}$ po qid & Inhibits fibrinolysis \\
\hline Aminocaproic acid & НHТ & 2 g po daily & Inhibits fibrinolytic system \\
\hline Desmopressin & HНT & IV & $\begin{array}{l}\text { Stabilizes vessel wall, } \\
\text { increased platelet adhesion }\end{array}$ \\
\hline Vasopressin & $A D$ & IV/IA & $\begin{array}{l}\text { Decreased splanchnic } \\
\text { circulation }\end{array}$ \\
\hline $\begin{array}{l}\text { Diamino-8-D-arginine } \\
\text { vasopressin }\end{array}$ & $A D+V W D$ & $\begin{array}{l}\text { IN spray, } 300 \\
\mu \mathrm{g}\end{array}$ & Increased vascular stability \\
\hline Thalidomide & AD, HHT & $\begin{array}{l}100 \mathrm{mg}-300 \\
\text { mg qid } 400 \\
\text { mg po daily }\end{array}$ & Antiangiogenesis \\
\hline
\end{tabular}

${ }^{\wedge}$ Reproduced from Can J Gastroenterol. 2006 Mar; 20(3): 171-180

AD Angiodysplasia; bid Two times per day; BRBNS Blue rubber bleb nevus syndrome; HHT Hereditary hemorrhagic telangiectasia; IA Intra-arterial; IM Intramuscular; IN Intranasal; IV Intravenous; PO Orally; QID Four times per day; SC Subcutaneous; TID Three times per day; VWD von Willebrand disease; WS Watermelon stomach

it has anti-angiogenetic effects. There are few case reports showing its positive impact in patients with refractory gastrointestinal (GI) bleeding secondary to arteriovenous malformations/angiodysplasias (AVM/AD). Patients showed improvement within 2 weeks which was sustained for more than 2.5 years with only minimal side effects of fatigue and transient peripheral neuropathy ${ }^{[31]-[34]}$.

\section{Conclusion}

While research continues to strive in innovating new strategies to prevent cardio-embolic phenomena in atrial fibrillation patients with minimal complications, in future, we should not lose the focus of atrial fibrillation (AF) patients at present. Roughly around $50 \%$ of atrial fibrillation (AF) patients with guideline indications for OAT end up not being on any kind of anticoagulation, which is of huge concern as stroke is the leading cause of disability and third leading cause of death in United States with an estimated annual cost of around 60 billion dollars. Bleeding complication remains one of the most common reasons for a patient or a clinician to discontinue warfarin and NOAC's are no better when it comes to bleeding events. We can approach this problem by actively addressing the source of bleeding and then coming up with a long-term strategy for stroke prevention. Systemic Octreotide can be reasonable option as a bridge to a more definitive therapy for stroke prevention.

\section{Conflict Of Interests}

$\mathrm{DL}$ - is the principle investigator for the Amaze Trial and Amulet IDE trial. He is also a consultant for St. Jude Medical 
Disclosures

None.

\section{References}

1. Lamassa M, Di CarloA, PracucciG, BasileA M, TrefoloniG, VanniP, SpolveriS, BaruffiM C, LandiniG, GhettiA, WolfeC D, InzitariD. Characteristics, outcome, and care of stroke associated with atrial fibrillation in Europe: data from a multicenter multinational hospital-based registry (The European Community Stroke Project). Stroke. 2001;32 (2):392-8

2. Lin HJ,WolfP A, Kelly-HayesM, BeiserA S, KaseC S, BenjaminE J, D'AgostinoR B. Stroke severity in atrial fibrillation. The Framingham Study. Stroke. 1996;27 (10):1760-4

3. Miyasaka Yoko, BarnesMarion E, GershBernard J, ChaStephen S, BaileyKent R, AbhayaratnaWalter P, SewardJames B, TsangTeresa S M. Secular trends in incidence of atrial fibrillation in Olmsted County, Minnesota, 1980 to 2000, and implications on the projections for future prevalence. Circulation. 2006;114 (2):119-25

4. Turagam Mohit K, ParikhValay, AfzalMuhammad R, GopinathannairRakesh, LavuMadhav, KanmanthareddyArun, PillarisettiJayasree, ReddyMadhu, AtkinsDonita, BommanaSudharani, JaegerMelissa, JefferyCourtney, MohantySanghamitra, SantangeliPasquale, ChengJie, Di BiaseLuigi, NarasimhanCalambur, NataleAndrea, LakkireddyDhanunjaya. Replacing warfarin with a novel oral anticoagulant: Risk of recurrent bleeding and stroke in patients with warfarin ineligible or failure in patients with atrial fibrillation (The ROAR study). J. Cardiovasc. Electrophysiol. 2017;28 (8):853-861

5. Kachroo Sumesh, HamiltonMelissa, LiuXianchen, PanXianying, BrixnerDiana, MarroucheNassir, BiskupiakJoseph. Oral anticoagulant discontinuation in patients with nonvalvular atrial fibrillation. Am J Manag Care. 2016;22 (1):e1-8

6. Thom Thomas, HaaseNancy, RosamondWayne, HowardVirginia J, RumsfeldJohn, ManolioTeri, ZhengZhi-Jie, FlegalKatherine, O'DonnellChristopher, KittnerSteven, Lloyd-JonesDonald, GoffDavid C, HongYuling, AdamsRobert, FridayGary, FurieKaren, GorelickPhilip, KisselaBrett, MarlerJohn, MeigsJames, RogerVeronique, SidneyStephen, SorliePaul, SteinbergerJulia, WasserthielSmollerSylvia, WilsonMatthew, WolfPhilip. Heart disease and stroke statistics--2006 update: a report from the American Heart Association Statistics Committee and Stroke Statistics Subcommittee. Circulation. 2006;113 (6):e85151

7. January Craig T, WannL Samuel, AlpertJoseph S, CalkinsHugh, CigarroaJoaquin E, ClevelandJoseph C, ContiJamie B, EllinorPatrick T, EzekowitzMichael D, FieldMichael E, MurrayKatherine T, SaccoRalph L, StevensonWilliam G, TchouPatrick J, TracyCynthia M, YancyClyde W. 2014 AHA/ACC/HRS guideline for the management of patients with atrial fibrillation: a report of the American College of Cardiology/American Heart Association Task Force on Practice Guidelines and the Heart Rhythm Society. J. Am. Coll. Cardiol. 2014;64 (21):e1-76

8. Hansen Morten L, SørensenRikke, ClausenMette T, Fog-PetersenMarie Louise, RaunsøJakob, GadsbøllNiels, GislasonGunnar H, FolkeFredrik, AndersenSøren S, SchrammTina K, AbildstrømSteen Z, PoulsenHenrik E, KøberLars, TorpPedersenChristian. Risk of bleeding with single, dual, or triple therapy with warfarin, aspirin, and clopidogrel in patients with atrial fibrillation. Arch. Intern. Med. 2010;170 (16):1433-41

9. Hylek Elaine M, GoAlan S, ChangYuchiao, JensvoldNancy G, HenaultLori E, SelbyJoe V, SingerDaniel E. Effect of intensity of oral anticoagulation on stroke severity and mortality in atrial fibrillation. N. Engl.J. Med. 2003;349 (11):1019-26

10. Adjusted-dose warfarin versus low-intensity, fixed-dose warfarin plus aspirin for high-risk patients with atrial fibrillation: Stroke Prevention in Atrial Fibrillation III randomised clinical trial. Lancet. 1996;348 (9028):633-8

11. Connolly S, PogueJ, HartR, PfefferM, HohnloserS, ChrolaviciusS, PfefferM, HohnloserS, YusufS. Clopidogrel plus aspirin versus oral anticoagulation for atrial fibrillation in the Atrial fibrillation Clopidogrel Trial with Irbesartan for prevention of Vascular Events (ACTIVE W): a randomised controlled trial. Lancet. 2006;367 (9526):1903-12

12. Bousser M G, BouthierJ, BüllerH R, CohenA T, CrijnsH, DavidsonB L, HalperinJ, HankeyG, LevyS, PengoV, PrandoniP, PrinsM H, TomkowskiW, Torp-PedersenC, Thorp-PedersenC, WyseD G. Comparison of idraparinux with vitamin $\mathrm{K}$ antagonists for prevention of thromboembolism in patients with atrial fibrillation: a randomised, open-label, non-inferiority trial. Lancet. 2008;371 (9609):315-21

13. Diener Hans-Christoph. Stroke prevention using the oral direct thrombin inhibitor ximelagatran in patients with non-valvular atrial fibrillation. Pooled analysis from the SPORTIF III and V studies. Cerebrovasc. Dis. 2006;21 (4):27993

14. Yao Xiaoxi, AbrahamNeena S, SangaralinghamLindsey R, BellolioM Fernanda, McBaneRobert D, ShahNilay D, NoseworthyPeter A. Effectiveness and Safety of Dabigatran, Rivaroxaban, and Apixaban Versus Warfarin in Nonvalvular Atrial Fibrillation. J Am Heart Assoc. 2016;5 (6)

15. Miller Corey S, GrandiSonia M, ShimonyAvi, FilionKristian B, EisenbergMark J. Meta-analysis of efficacy and safety of new oral anticoagulants (dabigatran, rivaroxaban, apixaban) versus warfarin in patients with atrial fibrillation. Am. J. Cardiol. 2012;110 (3):453-60

16. Holmes David R, DoshiShephal K, KarSaibal, PriceMatthew J, SanchezJose M, SievertHorst, ValderrabanoMiguel, ReddyVivek Y. Left Atrial Appendage Closure as an Alternative to Warfarin for Stroke Prevention in Atrial Fibrillation: A Patient-Level Meta-Analysis. J. Am. Coll. Cardiol. 2015;65 (24):2614-2623

17. Meschia James F, BushnellCheryl, Boden-AlbalaBernadette, BraunLynne T, BravataDawn M, ChaturvediSeemant, CreagerMark A, EckelRobert H, ElkindMitchell S V, FornageMyriam, GoldsteinLarry B, GreenbergSteven M, HorvathSusanna E, IadecolaCostantino, JauchEdward C, MooreWesley S, WilsonJohn A. Guidelines for the primary prevention of stroke: a statement for healthcare professionals from the American Heart Association/American Stroke Association. Stroke. 2014;45 (12):3754-832

18. Kirchhof Paulus, BenussiStefano, KotechaDipak, AhlssonAnders, AtarDan, CasadeiBarbara, CastelláManuel, DienerHans-Christoph, HeidbuchelHein, HendriksJeroen, HindricksGerhard, ManolisAntonis S, OldgrenJonas, Alexandru PopescuBogdan, SchottenUlrich, Van PutteBart, VardasPanagiotis. 2016 ESC Guidelines for the Management of Atrial Fibrillation Developed in Collaboration With EACTS. Rev Esp Cardiol (Engl Ed). 2017;70 (1)

19. Meier Bernhard, BlaauwYuri, KhattabAhmed A, LewalterTorsten, SievertHorst, TondoClaudio, GliksonMichael. EHRA/EAPCI expert consensus statement on catheter-based left atrial appendage occlusion. Europace. 2014;16 (10):1397-416

20. Raju Gottumukkala S, GersonLauren, DasAnanya, LewisBlair. American Gastroenterological Association (AGA) Institute medical position statement on obscure gastrointestinal bleeding. Gastroenterology. 2007;133 (5):1694-6

21. Parker Donna R, LuoXuemei, JalbertJessica J, AssafAnnlouise R. Impact of upper and lower gastrointestinal blood loss on healthcare utilization and costs: a systematic review. J Med Econ. 2011;14 (3):279-87

22. Sami S S, Al-ArajiS A, RagunathK. Review article: gastrointestinal angiodysplasia - pathogenesis, diagnosis and management. Aliment. Pharmacol. Ther. 2014;39 (1):15-34

23. Brown Colin, SubramanianVenkataraman, WilcoxC Mel, PeterShajan. Somatostatin analogues in the treatment of recurrent bleeding from gastrointestinal vascular malformations: an overview and systematic review of prospective observational studies. Dig. Dis. Sci. 2010;55 (8):2129-34

24. Bon C, AparicioT, VincentM, MavrosM, BejouB, RaynaudJ-J, ZampeliE, AirineiG, SautereauD, BenamouzigR, MichopoulosS. Long-acting somatostatin analogues decrease blood transfusion requirements in patients with refractory gastrointestinal bleeding associated with angiodysplasia. Aliment. Pharmacol. 
Ther. 2012;36 (6):587-93

25. Vase P. Estrogen treatment of hereditary hemorrhagic telangiectasia. A doubleblind controlled clinical trial. Acta Med Scand. 1981;209 (5):393-6

26. Moshkowitz M, ArberN, AmirN, GilatT. Success of estrogen-progesterone therapy in long-standing bleeding gastrointestinal angiodysplasia. Report of a case. Dis. Colon Rectum. 1993;36 (2):194-6

27. Barkin J S, RossB S. Medical therapy for chronic gastrointestinal bleeding of obscure origin. Am. J. Gastroenterol. 1998;93 (8):1250-4

28. Bauditz Juergen. Effective treatment of gastrointestinal bleeding with thalidomide-Chances and limitations. World J. Gastroenterol. 2016;22 (11):3158-64

29. Junquera F, FeuF, PapoM, VidelaS, ArmengolJ R, BordasJ M, SaperasE, PiquéJ M, MalageladaJ R. A multicenter, randomized, clinical trial of hormonal therapy in the prevention of rebleeding from gastrointestinal angiodysplasia. Gastroenterology. 2001;121 (5):1073-9

30. Madanick Ryan D, BarkinJamie S. Hormonal therapy in angiodysplasia: should we completely abandon its use?. Gastroenterology. 2002;123 (6):2156; author reply 2156-7

31. Bauditz J, SchachschalG, WedelS, LochsH. Thalidomide for treatment of severe intestinal bleeding. Gut. 2004;53 (4):609-12

32. Shurafa M, KambojG. Thalidomide for the treatment of bleeding angiodysplasias. Am J Gastroenterol. 2003;98:221-222

33. Pérez-Encinas Manuel, Rabuñal MartínezMaria José, Bello LópezJosé Luis. Is thalidomide effective for the treatment of gastrointestinal bleeding in hereditary hemorrhagic telangiectasia?. Haematologica. 2002;87 (8)

34. Meijer K, PetersFT, van der MeerJ. Recurrent severe bleeding from gastrointestinal angiodysplasia in a patient with von Willebrand's disease, controlled with recombinant factor VIIa. Blood Coagul. Fibrinolysis. 2001;12 (3):211-3 